\title{
Advantages of Monochromated Low Voltage Aberration Corrected TEM
}

\author{
David C. Bell ${ }^{1}$, Chris Russo ${ }^{1}$, Stefan Meyer $^{2}$, Gerd Benner ${ }^{2}$ and Max Haider ${ }^{3}$ \\ 1. School of Engineering and Applied Sciences, and Center for Nanoscale Systems, \\ Harvard University, Cambridge, Massachusetts 02138 \\ 2. Carl Zeiss NTS GmbH, Carl Zeiss SMT, Oberkochen Germany \\ 3. CEOS GmbH, Heidelberg Germany
}

\begin{abstract}
Aberration Corrected Transmission Electron Microscopy (Cs TEM) has shown may benefits[1]; perhaps the greatest improvement in imaging performance has been in the lower voltage range (80-120 KeV). With modern Cs corrected TEM's able to obtain Angstrom level resolution at $80 \mathrm{KeV}$ the opens up the ability to investigate a wide range of materials problems. The combination of Cs correction and a source monochromator yields a further step along the path to an ideal microscope, the expansion of the temporal envelope function of the contrast transfer function by reducing the delta $\mathrm{E}$ is a direct benefit that can lead to improved resolution. The further benefits of monochromator integration are for applications that especially depend on energy resolution such as for spectroscopy and advanced contrast mechanisms such as "atomic scale" energy filtered imaging. The Schottky FEG shows a typical energy spread of $0.6 \mathrm{eV}$ and cold FEG's down to the $0.3 \mathrm{eV}$ region. The competing needs for high brightness, cost and stability have meant that cold FEG's are not as commonly available from microscope vendors as the thermal FEG, as well as the issues of stability, vacuum requirements. In the development process to produce an electron source with all the benefits of both cold and thermal emitters the incorporation of a monochromator on a thermal source is one of the mechanisms of choice to minimize the energy spread. The energy spread of the field emission source was reduced by the Zeiss/CEOS monochromator to $0.1 \mathrm{eV}$ at $200 \mathrm{KV}$ and $0.07 \mathrm{eV}$ at $80 \mathrm{KV}$ with suitable remaining brightness for high-resolution imaging.
\end{abstract}

The advantages of low voltage TEM imaging have been known for many years with reduced sample damage and typically improved contrast. These instruments will allow us insight in materials classes previously excluded from high-resolution analysis due to their beam-sensitivity. This will include light element materials which can now be investigated at an unprecedented contrast and spatial resolution. The further advantages of the addition of the in-column energy filter allows for the ability to image only the elastically scattered electrons, for HRTEM imaging (or the inelastically scattered if needed) and provides an additional mechanism for contrast.

Tunable Cs correction, monochromator and in-column energy filtering combined on one instrument platform provide a foundation for the future with a high resolution transmission electron microscope with advanced resolution and contrast capabilities.

\section{References}

[1] P.W. Hawkes and J.C.H. Spence Science of Microscopy Springer (2007) 

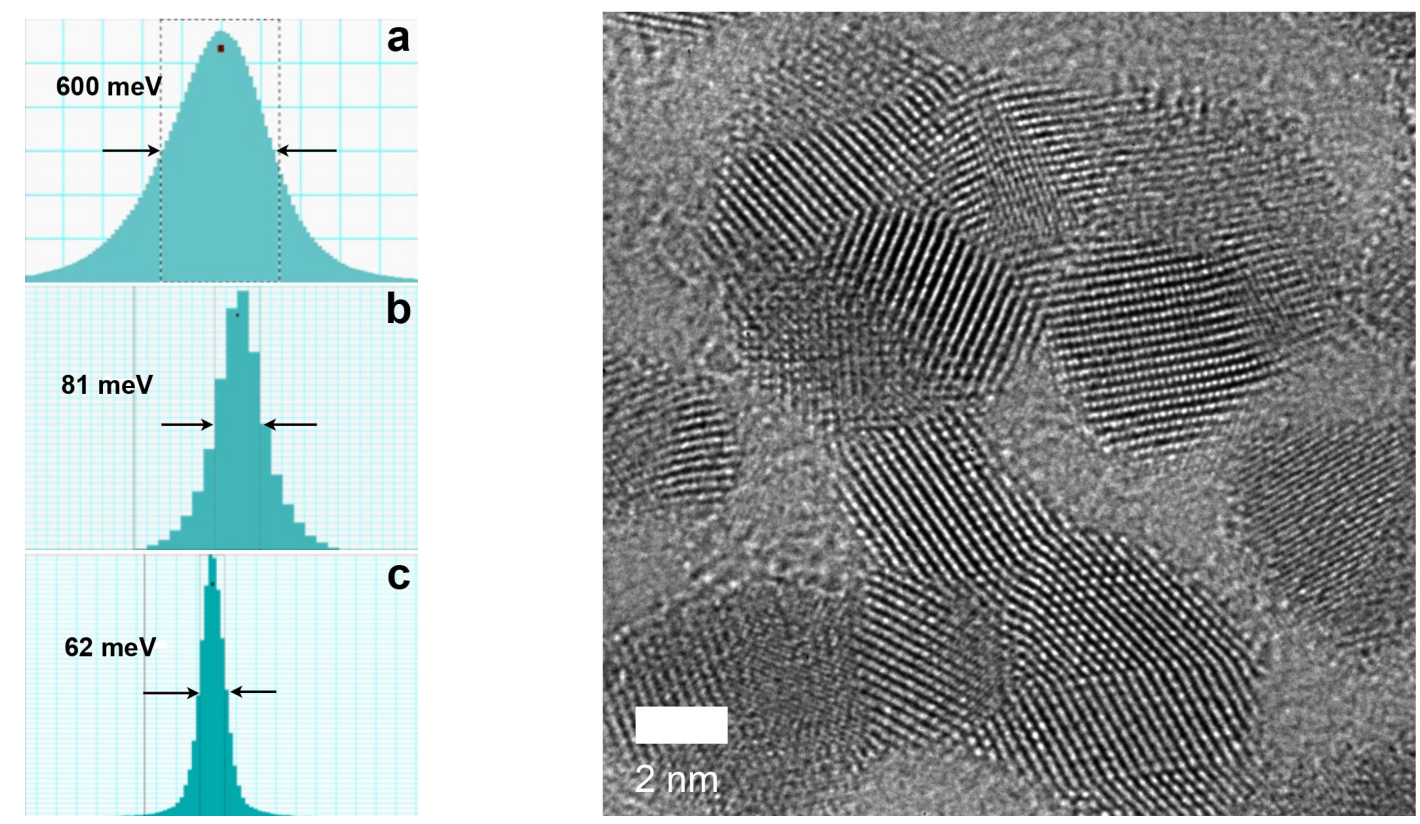

Figure 1. Left, Zero-loss peak showing energy spread with Zeiss-CEOS monochromator, a) non-monochromated b) monochromated (1um slit) at $200 \mathrm{KeV} \mathrm{c}$ ) monochromated (1um slit) at $80 \mathrm{KeV}$. Right, Monochromated Cs-corrected image of Pt particles on carbon film.
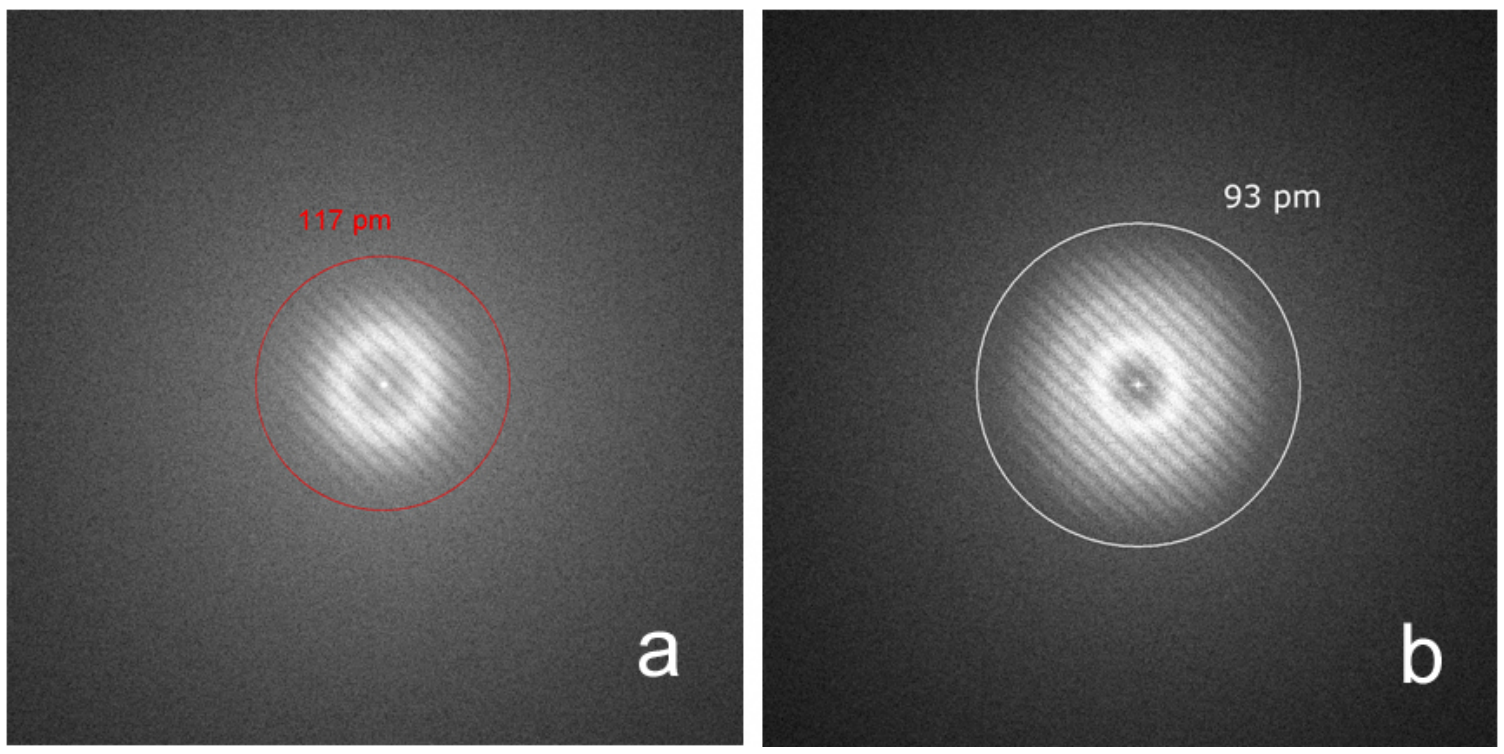

Figure 2. At $80 \mathrm{KeV}$. a) Young's fringes produced from ultra thin Tungsten film with Cs correction showing information transfer to slightly less than $0.1 \mathrm{~nm}$. b) Young's fringes produced with the same Cs correction but with monochromation of $0.2 \mathrm{eV}$ energy spread. 